\title{
Transition Form Factor in Higgs Production through Two-photon Processes
}

Norihisa Watanabe

High Energy Accelerator Research Organization (KEK)

Tsukuba, Ibaraki 305-0801, Japan

E-mail: norihisa@post.kek.jp

\section{Yoshimasa Kurihara}

High Energy Accelerator Research Organization (KEK)

Tsukuba, Ibaraki 305-0801, Japan

E-mail: kuriharaepost.kek.jp

\section{Ken Sasaki}

Dept. of Physics, Faculty of Engineering, Yokohama National University

Yokohama 240-8501, Japan

E-mail: sasaki@ynu.ac.jp

\section{Tsuneo Uematsu*}

Institute for Liberal Arts and Sciences, Kyoto University, Yoshida, Kyoto 606-8501, Japan and Maskawa Institute, Kyoto Sangyo University, Kyoto 603-8555,Japan

E-mail: uematsuescphys.kyoto-u.ac.jp

\begin{abstract}
We investigate the Higgs production in the two-photon fusion process realized in either electronpositron or electron-photon collision where one of the photons is off-shell while the other one is on-shell. We particularly interested in the case where the scattered electron or positron is detected (single tagging) which is described by the so-called transition form factor. The contributions to the transition form factor coming from top-quark loops as well as W-boson loops are evaluated. The $Q^{2}$ dependence of each contribution as well as the differential cross section for the Higgs production are numerically studied.
\end{abstract}

11th International Symposium on Radiative Corrections (Applications of Quantum Field Theory to Phenomenology) (RADCOR 2013),

22-27 September 2013

Lumley Castle Hotel, Durham, UK

\footnotetext{
${ }^{*}$ Speaker.
} 


\section{Introduction}

Recently there has been much interest in the diphoton decay of the Higgs boson discovered at LHC experiments [1], since its coupling to the photon is connected with the question whether it is really the one within the Standard Model (SM) or the one beyond SM, such as a supersymmetric extension of SM (MSSM) and its generalization or in composite models. It would be intriguing to investigate the properties of the Higgs particle through the production process in the two-photon fusion: $2 \gamma \rightarrow H$, which might be realized at ILC [2] and is just the opposite reaction of the diphoton decay mode of the Higgs particle: $H \rightarrow 2 \gamma$. The Higgs decay goes through the top-quark loops and the W-boson loops as discussed in Refs. [3, 4, 5, 6, 7, 8, 9] and the references therein.

Here we particularly interested in the two-photon process of the electron-positron collision in Fig.1(a) where one of the scattered electron (or positron) is detected (single tagging) [10]. From this process we can measure the so-called "transition form factor" of the Higgs particle as a function of the virtual-photon mass squared. Note that the $\gamma^{*} \gamma \rightarrow \pi^{0}$ transition form factor was theoretically investigated in QCD [11]. The recent experimental data were given in Refs. [12, 13].

As is well-known the leading processes for the Higgs production in $e^{+} e^{-}$collision at the tree level are the s-channel Higgs-strahlung $\left(e^{+} e^{-} \rightarrow Z^{*} \rightarrow Z H\right), W^{+} W^{-}$fusion $\left(e^{+} e^{-} \rightarrow v_{e} \bar{v}_{e} H\right)$ and $Z Z$ fusion $\left(e^{+} e^{-} \rightarrow e^{-} e^{+} H\right)$. The first two processes are excluded by performing the single tagging of the scattered electron (or positron), only leaving $Z Z$ fusion process.

So let us consider for the moment the two-photon fusion process in $e \gamma$ collision illustrated in Fig.1(b) to avoid the $Z Z$ fusion contribution.

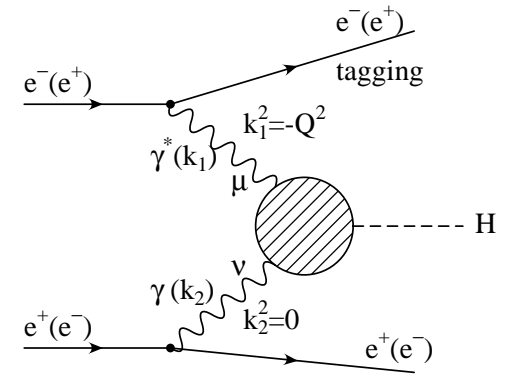

(a)

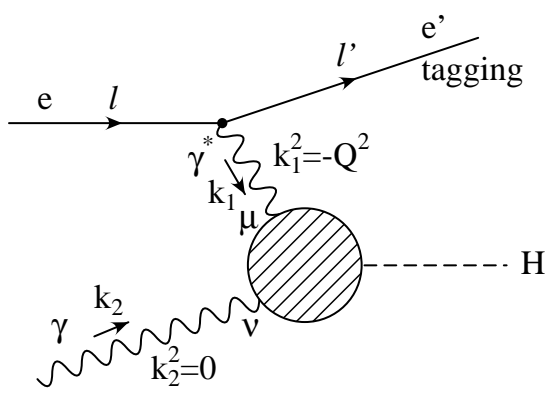

(b)

Figure 1: Two-photon fusion process in (a) $e^{+} e^{-}$collision and (b) $e \gamma$ collision for the Higgs production.

We first examine the tensor structure of the transition amplitude for the fusion of the virtual and real photons into the Higgs particle satisfying the gauge invariance. We then evaluate the contributions from the top-quark loops as well as from the W-boson loops to the Higgs production in the two-photon process [14].

\section{Transition form factor}

Let us consider the scattering of electron off real photon for the Higgs production as depicted in Fig.1(b):

$$
e(l)+\gamma\left(k_{2}\right) \rightarrow e^{\prime}\left(l^{\prime}\right)+H\left(k_{1}+k_{2}\right)
$$


where the scattering amplitude is given by

$$
\left\langle e^{\prime} H|T| e \gamma\right\rangle=\bar{u}\left(l^{\prime}\right)\left(-i e \gamma^{\mu}\right) u(l) \frac{-i}{k_{1}^{2}+i \varepsilon} A_{\mu \nu} \varepsilon^{v}\left(k_{2}\right)
$$

The transition amplitude for $\gamma^{*} \gamma \rightarrow H$ extracted from the $e \gamma$ collision shown in Fig.2 reads

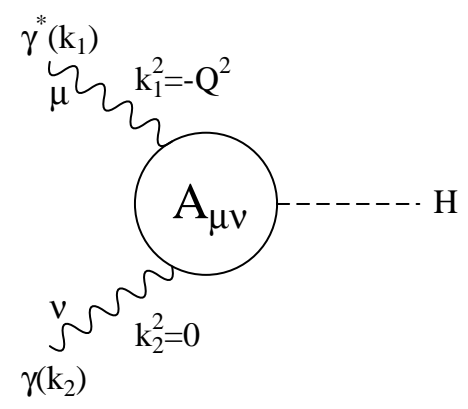

Figure 2: The transition amplitude for Higgs production via virtual and real photon fusion.

$$
M \equiv\left\langle H(q)|T| \gamma^{*}\left(k_{1}\right) \gamma\left(k_{2}\right)\right\rangle=\varepsilon^{\mu}\left(k_{1}\right) \varepsilon^{v}\left(k_{2}\right) A_{\mu v}\left(k_{1}, k_{2}\right)
$$

where $\varepsilon^{\mu}\left(k_{1}\right)\left(\varepsilon^{v}\left(k_{2}\right)\right)$ is the polarization vector for the incident virtual (real) photon and we have introduced the tensor $A_{\mu \nu}$ which can be decomposed as

$$
\begin{array}{r}
A_{\mu v}\left(k_{1}, k_{2}\right)=\left(g_{\mu v}\left(k_{1} \cdot k_{2}\right)-k_{2 \mu} k_{1 v}\right) S_{1}\left(m^{2}, Q^{2}, m_{H}^{2}\right) \\
+\left(k_{1 \mu} k_{2 v}-\frac{k_{1}^{2}}{k_{1} \cdot k_{2}} k_{2 \mu} k_{2 v}\right) S_{2}\left(m^{2}, Q^{2}, m_{H}^{2}\right)
\end{array}
$$

where $k_{1}^{2}=-Q^{2}<0, k_{2}^{2}=0$ and $\left(k_{1}+k_{2}\right)^{2}=m_{H}^{2}$. We denote collectively the mass of the intermediate particle in the loop by $m$. Since $k_{2}^{v} \varepsilon_{v}\left(k_{2}\right)=0$, the transition amplitude reads

$$
M=\left[g_{\mu v}\left(k_{1} \cdot k_{2}\right)-k_{2 \mu} k_{1 v}\right] S_{1}\left(m^{2}, Q^{2}, m_{H}^{2}\right) \varepsilon^{\mu}\left(k_{1}\right) \varepsilon^{v}\left(k_{2}\right) .
$$

For real and virtual two-photon fusion, we define the transition form factor $F_{i}\left(m^{2}, Q^{2}, m_{H}^{2}\right)$ by

$$
F_{i}\left(m^{2}, Q^{2}, m_{H}^{2}\right)=S_{1}\left(m^{2}, Q^{2}, m_{H}^{2}\right) /\left(\frac{g e^{2}}{(4 \pi)^{2}} \frac{1}{m_{W}}\right)
$$

where $i=1 / 2,1$ for a fermion-loop $F_{1 / 2}$ and for the W-boson loop $F_{1}$, respectively. $e$ and $g$ are the electromagnetic and weak gauge couplings, respectively, and $m_{W}$ is the $W$ boson mass.

The total transition form factor is given by

$$
F_{\text {total }}\left(Q^{2}, m_{H}^{2}\right)=\sum_{f} N_{c} e_{f}^{2} F_{1 / 2}\left(m_{f}^{2}, Q^{2}, m_{H}^{2}\right)+F_{1}\left(m_{W}^{2}, Q^{2}, m_{H}^{2}\right)
$$

where $N_{c}$ is the number of the colors ( 1 for leptons and 3 for quarks) and $e_{f}$ is the electric charge of the fermion $f$ in the unit of proton charge. 
Here we note that the transition form factor $F_{i}$ shows a scaling behavior:

$$
F_{i}\left(m^{2}, Q^{2}, m_{H}^{2}\right) \rightarrow F_{i}(\rho, \tau),
$$

where $m$ is the mass of the particle in the loop, i.e. $m=m_{f} \quad(f=u, d, s, c, b, t)$ or $m=m_{W}$ and

$$
\rho_{f} \equiv \frac{Q^{2}}{4 m_{f}^{2}}, \quad \tau_{f} \equiv \frac{4 m_{f}^{2}}{m_{H}^{2}}, \quad \rho_{W} \equiv \frac{Q^{2}}{4 m_{W}^{2}}, \quad \tau_{W} \equiv \frac{4 m_{W}^{2}}{m_{H}^{2}} .
$$

\subsection{Fermion-loop contribution}
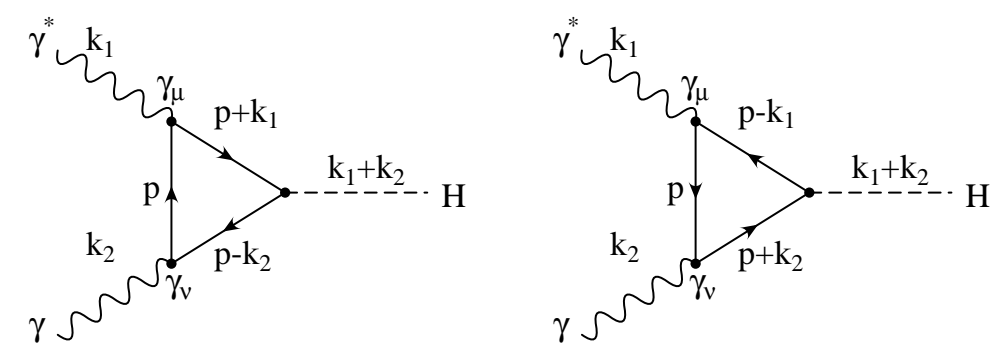

Figure 3: Fermion triangle-loop diagrams contributing to $\gamma^{*} \gamma \rightarrow H$

We calculate the contribution to the transition form factor of Higgs arising from charged fermion triangle-loop diagrams shown in Fig. 3 and obtain

$$
\begin{gathered}
F_{1 / 2}(\rho, \tau)=-\frac{2 \tau}{1+\rho \tau}\left\{1+\left(1-\frac{\tau}{1+\rho \tau}\right)\left(f(\tau)+\frac{1}{4} g(\rho)\right)\right. \\
\left.+\frac{\tau}{1+\rho \tau}(2 \rho \sqrt{\tau-1} \sqrt{f(\tau)}-\sqrt{\rho(\rho+1)} \sqrt{g(\rho)})\right\}
\end{gathered}
$$

where

$$
\begin{aligned}
f(\tau) & =\left[\sin ^{-1} \sqrt{\frac{1}{\tau}}\right]^{2}, \text { for } \tau \geq 1 \\
& =-\frac{1}{4}\left[\log \frac{1+\sqrt{1-\tau}}{1-\sqrt{1-\tau}}-i \pi\right]^{2} \text { for } \tau<1 \\
g(\rho) & =\left[\log \frac{\sqrt{\rho+1}+\sqrt{\rho}}{\sqrt{\rho+1}-\sqrt{\rho}}\right]^{2} .
\end{aligned}
$$

Eq.(2.10) shows that the fermion loop contribution $F_{1 / 2}$ is proportional to $\tau_{f}$, i.e., the fermion mass squared $m_{f}^{2}$. Thus the contributions to the transition form factor from leptons and light-flavour $(u, d, s, c$ and $b)$ quarks are negligibly small compared to the one from top quark. Therefore, from now on, we consider only the top quark loop contribution for $F_{1 / 2}$.

In the $Q^{2} \rightarrow 0$ limit (or $\rho \rightarrow 0$ limit) it reduces to the functions $F_{1 / 2}(\tau)$ appearing in the $H \rightarrow 2 \gamma$ decay-rate expression $[3,4,5,6,7,8,9]$ :

$$
F_{1 / 2}(\rho \rightarrow 0, \tau)=F_{1 / 2}(\tau)=-2 \tau[1+(1-\tau) f(\tau)] .
$$




\subsection{W-boson loop contribution}

Next we calculate the W-boson loop diagrams in unitary gauge shown in Fig.4 and obtain

$$
\begin{aligned}
& F_{1}(\rho, \tau) \\
& =\frac{1}{1+\rho \tau}\left\{\frac{\tau}{1+\rho \tau}\left(4 \rho+8 \rho^{2} \tau+6(1+\rho \tau)-3 \tau\right)\left(f(\tau)+\frac{1}{4} g(\rho)\right)\right. \\
& \quad+(4 \rho+2(1+\rho \tau)+3 \tau) \\
& \left.\quad \times\left(1+\frac{2 \rho \tau}{1+\rho \tau} \sqrt{\tau-1} \sqrt{f(\tau)}-\frac{\tau}{1+\rho \tau} \sqrt{\rho(\rho+1)} \sqrt{g(\rho)}\right)\right\}
\end{aligned}
$$

where the expressions of $f(\tau)$ and $g(\rho)$ are given in Eqs.(2.11) and (2.13), respectively.
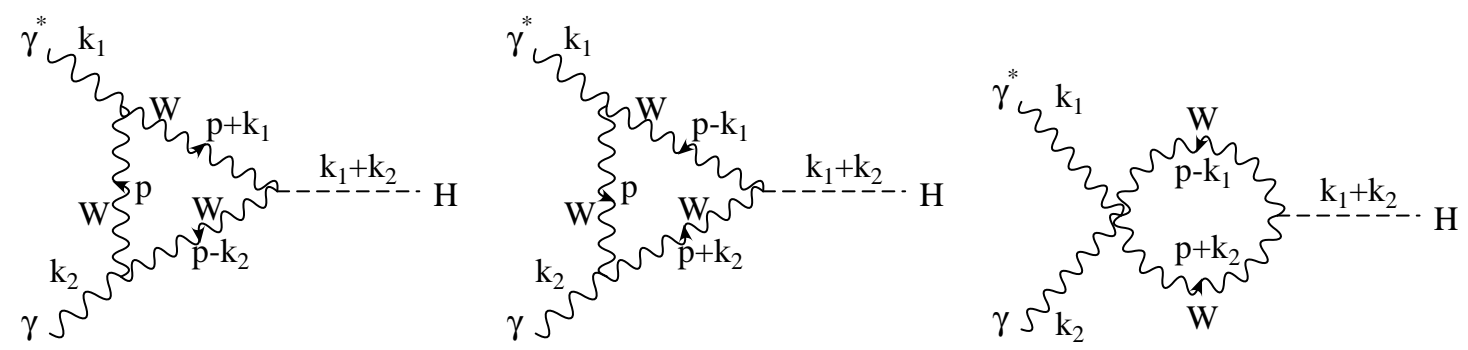

Figure 4: W-boson loop diagrams contributing to $\gamma^{*} \gamma \rightarrow H$

In the $Q^{2} \rightarrow 0$ limit (or $\rho \rightarrow 0$ limit) it reduces to the functions $F_{1}(\tau)$ which appears in the $H \rightarrow 2 \gamma$ decay-rate expression $[3,4,5,6,7,8,9]$ :

$$
F_{1}(\rho \rightarrow 0, \tau)=F_{1}(\tau)=2+3 \tau+3 \tau(2-\tau) f(\tau)
$$

It is noted that $f(\tau)$ and $g(\rho)$ appear in the expressions of $F_{1 / 2}(\rho, \tau)$ in Eq.(2.10) and $F_{1}(\rho, \tau)$ in Eq.(2.15) as the following combinations,

$$
f(\tau)+\frac{1}{4} g(\rho) \text { and } 2 \rho \sqrt{\tau-1} \sqrt{f(\tau)}-\sqrt{\rho(\rho+1)} \sqrt{g(\rho)},
$$

which come from two- and three-point scalar integrals [15], as will be discussed in a separate paper [16].

\section{Numerical analysis}

Based on the results obtained in the previous section we now numerically investigate the behavior of the transition form factor. Any deviation from its $Q^{2}$ dependence in the Standard Model (SM) might indicate a possible signature of the new physics beyond the SM, such as MSSM [17] or composite models $[18,19]$.

In the large $Q^{2}$ limit, the two form factors, $F_{1 / 2}, F_{1}$ show quite different behaviors:

$$
\begin{aligned}
& F_{1 / 2}(\rho \rightarrow \infty, \tau: \text { fixed })=-\frac{1}{2 \rho} g(\rho)=-\frac{2 m_{t}^{2}}{Q^{2}} \log ^{2} \frac{Q^{2}}{m_{t}^{2}} \\
& F_{1}(\rho \rightarrow \infty, \tau: \text { fixed })=2 g(\rho)=2 \log ^{2} \frac{Q^{2}}{m_{W}^{2}}
\end{aligned}
$$




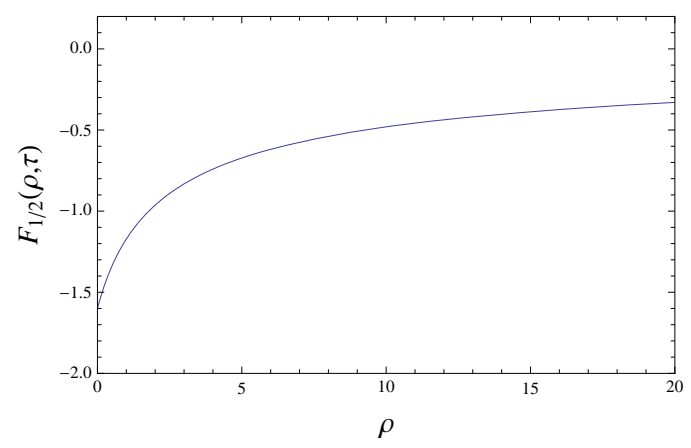

(a)

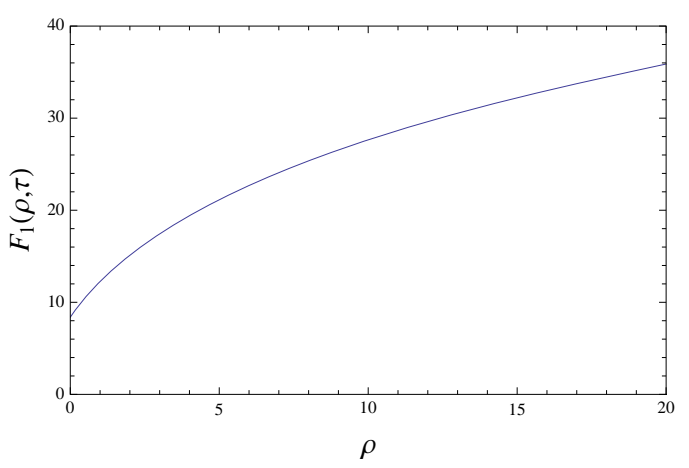

(b)

Figure 5: Transition form factor as a function of $\rho$, (a) $F_{1 / 2}$ the top-quark loop contribution, for $\tau=7.54$ and (b) $F_{1}$ the $W$-boson loop contribution $\tau=1.61$.

where we note that $g(\rho) \rightarrow \log ^{2}(4 \rho)$ as $\rho \rightarrow \infty$. Thus $F_{1 / 2}$ is decreasing to zero while $F_{1}$ is ever increasing as shown in Fig.5 (a) and (b). Taking the mass parameters to the values $m_{H}=126 \mathrm{GeV}$, $m_{t}=173 \mathrm{GeV}$ and $m_{W}=80 \mathrm{GeV}$, here we have plotted $F_{1 / 2}(\rho, \tau)\left(F_{1}(\rho, \tau)\right)$ with fixed $\tau=7.54$ $(\tau=1.61)$, as a function of $\rho=Q^{2} /\left(4 m_{t}^{2}\right)\left(\rho=Q^{2} /\left(4 m_{W}^{2}\right)\right)$.

In Fig.6 we have shown the $Q^{2}$ dependence of the total transition form factor including the $\mathrm{W}$-boson and top-quark loop contributions. Note that the contributions from light-flavor $(u, d, s, c$ and $b$ ) quarks are negligible.

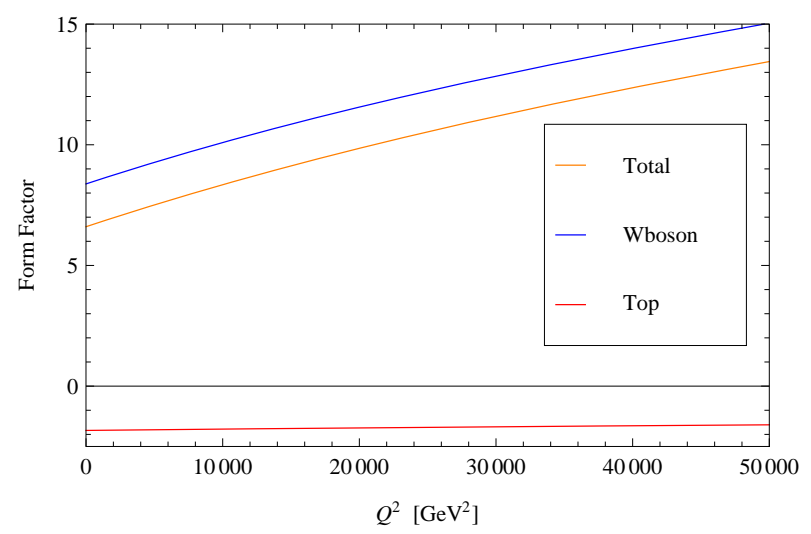

Figure 6: Transition form factor as a function $Q^{2}$. Red, blue and orange curves correspond to top-quark, W-boson and total contributions, respectively. We choose mass parameters as $m_{H}=126 \mathrm{GeV}, m_{t}=173 \mathrm{GeV}$ and $m_{W}=80 \mathrm{GeV}$.

The differential cross section for the Higgs production via $\gamma^{*} \gamma$ fusion in $e \gamma \rightarrow e H$ shown in Fig.1(b) is given by

$$
\frac{d \sigma_{\left(\gamma^{*} \gamma \text { fusion }\right)}}{d Q^{2}}=\frac{\alpha_{\mathrm{em}}^{3}}{64 \pi} \frac{g^{2}}{4 \pi} \frac{1}{Q^{2}}\left[1+\frac{u^{2}}{s^{2}}\right] \frac{1}{m_{W}^{2}}\left|F_{\text {total }}\left(Q^{2}\right)\right|^{2},
$$

where $s=\left(l+k_{2}\right)^{2}, u=\left(k_{2}-l^{\prime}\right)^{2}$ and $\alpha_{\mathrm{em}}=e^{2} /(4 \pi)$. 


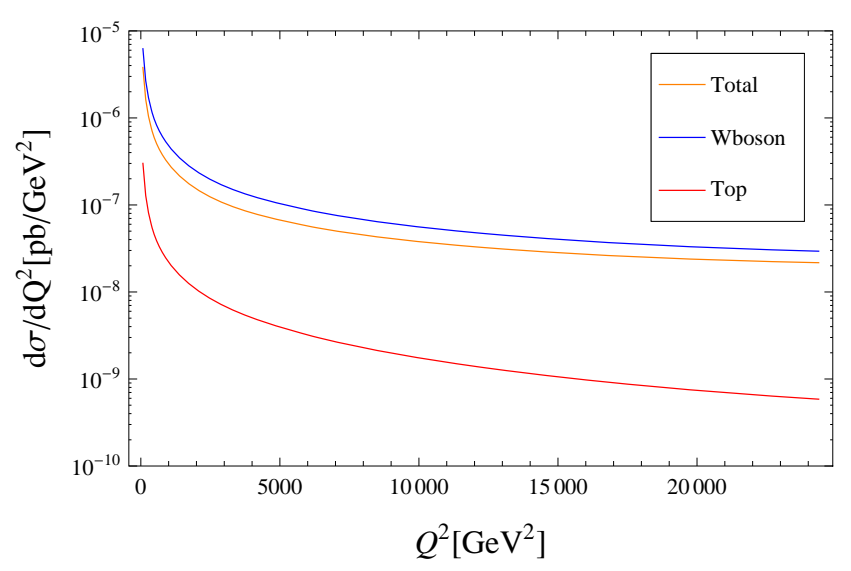

Figure 7: The differential cross section for the Higgs production as a function of $Q^{2}$. Red, blue and orange curves correspond to top-quark, W-boson and total contributions, respectively.

We have plotted the differential cross section $d \sigma / d Q^{2}$ as a function of $Q^{2}$ for $\sqrt{s}=200 \mathrm{GeV}$ in Fig.7. Here we note that the W-boson contribution (orange curve) dominates over the top-quark contribution (red curve) for the whole $Q^{2}$ region.

The possible competing process for $e+\gamma \rightarrow e^{\prime}+H$ with single electron tagging is $Z \gamma$ fusion process which is obtained in Fig.1(b) by replacing the virtual photon $\gamma^{*}$ with the Z-boson. Because of the mass squared term in the Z-boson propagator, as well as the $Q^{2}$ which appears as an overall factor, we find that the $\gamma^{*} \gamma$ fusion gives rise to the dominant contribution for the forward region, where $Q^{2}$ is smaller than $(100)^{2} \mathrm{GeV}^{2}$. In addition, regarding the total cross section, $\gamma^{*} \gamma$ fusion is dominant over $Z \gamma$ fusion process for $\sqrt{s} \leq 400 \mathrm{GeV}$, which will be discussed in the Ref. [16].

\section{Conclusion}

In this paper we have studied the transition form factor of SM Higgs particle which is coming from the top-quark loops as well as from the W-boson loops. The $Q^{2}$ dependence of each contribution is described in Figs. 5 and 6. It has turned out that the W-boson loop contribution dominates over the top-quark loop contribution. And we also found that the contribution from the leptons, light-flavor (u,d,c,s,b) quarks and from the possible charged scalars are negligible [14]. The differential cross section due to these transition form factors are also studied.

The transition form factor of the Higgs boson may also be measured in the electron-positron collision with single tagging, where $Z Z$ fusion process might contribute. However, if the kinematical region is restricted to the forward directions, $Z Z$ fusion is expected to be insignificant compared to $\gamma^{*} \gamma$ fusion $[14,16]$. Then using the equivalent-photon method, the so-called WeizsäckerWilliams method in $e^{+} e^{-}$collision [20], the corresponding Higgs production cross section can be written in terms of the transition form factor given in Eq. (2.6).

Finally, it would be interesting to see if the transition form factor is a useful ingredient for studying the Higgs physics at the photon collider discussed in Refs. [21, 22, 23]. As for a further subject we should include the higher-order effects due to QCD and Electro-Weak interactions. 


\section{Acknowledgments}

We would like to thank the organizers of the RADCOR 2013 for the hospitality at such a well-organized and stimulating symposium.

\section{References}

[1] ATLAS Collaboration, Phys. Lett. B 716 (2012) 1-29; CMS Collaboration, Phys. Lett. B 716 (2012) 30-61.

[2] http://www.linearcollider.org/cms.

[3] J. Ellis, M. K. Gaillard and D. V. Nanopoulos, Nucl. Phys. B 106 (1976) 292.

[4] B. L. Ioffe and V. A. Khoze, Fiz. Elem.I Chastits At. Yadra 9 (1978) 118 [Sov. J. Part. Nucl. 9 (1978) $50]$.

[5] M. A. Shifman, A. I. Vainshtein, M. B. Voloshin and V. I. Zakharov, Sov. J. Nucl. Phys. 30 (1979) 711 [Sov. J. Nucl. Phys. 30 (1979) 711]; Phys. Rev. D 85 (2012) 013015.

[6] T. G. Rizzo, Phys. Rev. D 22 (1980) 178.

[7] M. B. Gavela, G. Girardi, C. Malleville and P. Sorba, Nucl. Phys. B 193 (1981) 257.

[8] W. J. Marciano, C. Zhang and S. Willenbrock, Phys. Rev. D 85 (2012) 013002.

[9] J. F. Gunion, H. E. Haber, G. Kane and S. Dawason, “The Higgs Hunter's Guide”(Addison-Wesley, 1990).

[10] T. F. Walsh, Phys. Lett. B 36 (1971) 121; S. J. Brodsky, T. Kinoshita and H. Terazawa, Phys. Rev. Lett. 27 (1971) 280.

[11] G. P. Lepage and S. J. Brodsky, Phys. Rev. D 9 (1980) 2157.

[12] B. Aubert et al., (The BaBar Collaboration), Phys. Rev. D 80 (2009) 052002.

[13] S. Uehara et al., (The Belle Collaboration), Phys. Rev. D 86 (2012) 092007.

[14] N. Watanabe, Y. Kurihara, K. Sasaki and T. Uematsu, arXiv:1311.1601 [hep-ph] (2013).

[15] G. Passarino and M. Veltman, Nucl. Phys. B 160 (1979) 151.

[16] K. Sasaki, these proceedings. N. Watanabe, Y. Kurihara, T. Uematsu and K. Sasaki, in preparation.

[17] H. E. Haber and G. L. Kane, Phys. Rep. 117 (1985) 75.

[18] E. Farhi and L. Susskind, Phys. Rep. 74 (1981) 277.

[19] D. B. Kaplan, H. Georgi and S. Dimopoulos, Phys. Lett. B 136 (1984) 183.

[20] S. J. Brodsky, T. Kinoshita and H. Terazawa, Phys. Rev. Lett. 27 (1971) 280; Phys. Rev. D 4 (1971) 1532.

[21] M. M. Mühlleitner, M. Krämer, M. Spira and P. M. Zerwas, Phys. Lett. B 508 (2001) 311.

[22] M. M. Mühlleitner, Talk at the Photon Collider Workshop 2005, Kazimierz, Poland. arXiv:hep-ph/0512232(2005).

[23] I. F. Ginzburg and M. Krawczyk, "Testing Higgs Physics at the Photon Collider, [arXiv:1310.5881[hep-ph]]. 